\title{
In vitro activity of BAL30072 against Burkholderia pseudomallei
}

\author{
Takehiko Mima $^{\mathrm{a}, 1}$, Brian H. Kvitko ${ }^{\mathrm{a}}$, Drew A. Rholl ${ }^{\mathrm{a}}$, Malcolm G.P. Page ${ }^{\mathrm{b}}$, Eric Desarbre ${ }^{\mathrm{b}}$, \\ and Herbert P. Schweizer ${ }^{a,{ }^{*}}$
}

aDepartment of Microbiology, Immunology and Pathology, Rocky Mountain Regional Center of Excellence for Biodefense and Emerging Infectious Diseases Research, Colorado State University, Fort Collins, CO 80523-0922, USA bBasilea Pharmaceutica International Ltd., $\mathrm{CH}-4005$ Basel, Switzerland

\begin{abstract}
Burkholderia pseudomalle $i$ is an intrinsically antibiotic-resistant Category B priority pathogen and the aetiological agent of melioidosis. Treatment of $B$. pseudomallei infection is biphasic and lengthy in order to combat the acute and chronic phases of the disease. Acute-phase treatment preferably involves an intravenous cephalosporin (ceftazidime) or a carbapenem (imipenem or meropenem). In this study, the anti-B. pseudomallei efficacy of a new monosulfactam, BAL30072, was tested against laboratory strains $1026 \mathrm{~b}$ and $1710 \mathrm{~b}$ and several isogenic mutant derivatives as well as a collection of clinical and environmental B. pseudomallei strains from Thailand. More than $93 \%$ of the isolates had minimal inhibitory concentrations (MICs) in the range 0.004-0.016 $\mu \mathrm{g} / \mathrm{mL}$. For the laboratory strain 1026b, the MIC of BAL30072 was $0.008 \mu \mathrm{g} / \mathrm{mL}$, comparable with the MICs of $1.5 \mu \mathrm{g} / \mathrm{mL}$ for ceftazidime, $0.5 \mu \mathrm{g} / \mathrm{mL}$ for imipenem and $1 \mu \mathrm{g} / \mathrm{mL}$ for meropenem. Time-kill curves revealed that BAL30072 was rapidly bactericidal, killing $>99 \%$ of bacteria in $2 \mathrm{~h}$. BAL30072 activity was not significantly affected by efflux, it was only a marginal substrate of PenA $\beta$-lactamase, and activity was independent of malleobactin production and transport and the ability to transport pyochelin. In summary, BAL30072 has superior in vitro activity against $B$. pseudomallei compared with ceftazidime, meropenem or imipenem and it is rapidly bactericidal.
\end{abstract}

\author{
Keywords \\ Burkholderia pseudomallei; Melioidosis; Therapy; Monosulfactam; Efflux; Siderophore
}

\section{Introduction}

Burkholderia pseudomallei is the aetiological agent of melioidosis, a rare but serious and emerging tropical disease [1]. In the USA, the bacterium is also a Category B priority and Select Agent pathogen because of its potential use as biological weapon [2]. The current treatment regimen for human melioidosis is intravenous administration of ceftazidime or a

\footnotetext{
*Corresponding author. Present address: Department of Microbiology, Immunology and Pathology, Colorado State University, IDRC at Foothills Campus, Campus Delivery 0922, Fort Collins, CO 80523-0922, USA. Tel.: +1 970491 3536; fax: +1 9704918708. Herbert.Schweizer@colostate.edu (H.P. Schweizer)..

${ }^{1}$ Present address: Department of Microbiology, Kitasato University Medical School, 1-15-1 Kitasato, Minami-ku, Sagamihara, Kanagawa 252-0374, Japan.

Competing interests HPS was supported by a grant-in-aid of research by Basilea Pharmaceutica International Ltd. MGPP and ED are employees of Basilea Pharmaceutica Ltd. All other authors declare no competing interests.
}

Ethical approval Not required. 
carbapenem (imipenem or meropenem) for at least 10-14 days, followed by oral eradication therapy using trimethoprim/sulfamethoxazole with or without doxycycline for 12-20 weeks $[3,4]$. For patients who cannot tolerate this therapy or where it is contraindicated (e.g. children and pregnant women), the choice of oral therapy is amoxicillin/clavulanic acid (AMC). In a quest to increase the arsenal of therapeutics for melioidosis, the anti-B. pseudomallei efficacy of a new monosulfactam BAL30072 (Fig. 1) was tested, which has shown especially potent in vitro activity against Gram-negative bacteria that have acquired the reputation of 'superbugs' because of their high levels of resistance to many of the currently marketed antibiotics [5,6].

\section{Materials and methods}

\subsection{Bacterial strains}

The B. pseudomallei laboratory strains as well as their isogenic derivatives used in this study are listed in Table 1 . The study also included an additional 30 clinical and 30 environmental isolates from Thailand. Strain 1026b was originally isolated from a patient in Ubon Ratchathani in Northeast Thailand and is one of the widely used laboratory prototype strains [7].

\subsection{Susceptibility testing}

Susceptibility was tested by determining minimal inhibitory concentrations (MICs). MICs for BAL30072 and carbenicillin were determined using the two-fold broth microdilution technique [8] with Difco ${ }^{\mathrm{TM}}$ Mueller-Hinton broth (Becton Dickinson, Franklin Lakes, NJ) as the growth medium. Etest strips (AB bioMérieux, Marcy l'Étoile, France) were used to determine MICs for ceftazidime, amoxicillin, AMC, imipenem and meropenem. For timekill curves, ca. $10^{6} \log$-phase cells (optical density at $600 \mathrm{~nm} 0.5-0.7$ ) of strain $1026 \mathrm{~b}$ was inoculated in Luria-Bertani Lennox (LB) broth (MO BIO Laboratories, Carlsbad, CA, USA) with and without BAL30072. The cultures were shaken at $37^{\circ} \mathrm{C}$, aliquots were removed at regular time intervals and viable bacteria were determined by plating 10 -fold serial dilutions on LB agar plates. Time-kill curves were performed at $1 \times(0.008 \mu \mathrm{g} / \mathrm{mL})$ and $4 \times(0.032 \mu \mathrm{g} /$ $\mathrm{mL})$ MIC.

\section{Results and discussion}

\subsection{Susceptibility of strain $1026 b$}

Susceptibility of the clinical isolate, and now commonly used laboratory strain, 1026b [7] to BAL30072 was initially tested and was compared with that of other $\beta$-lactams (Table 2). The MIC for BAL30072 was $0.008 \mu \mathrm{g} / \mathrm{mL}$, comparable with the MICs of $1.5 \mu \mathrm{g} / \mathrm{mL}$ for ceftazidime, $2 \mu \mathrm{g} / \mathrm{mL}$ for AMC, $0.5 \mu \mathrm{g} / \mathrm{mL}$ for imipenem and $1 \mu \mathrm{g} / \mathrm{mL}$ for meropenem, all of which are used or have been tested for treatment of human melioidosis. The in vitro activity of BAL30072 is thus superior to these clinically used antibiotics. Time-kill curves revealed that BAL30072 was rapidly bactericidal, killing $>99 \%$ of bacteria in $2 \mathrm{~h}$ (data not shown).

\subsection{Susceptibility of clinical and environmental strains}

Next, the susceptibility of a panel of randomly chosen 30 clinical (plus strain 1026b) and 30 environmental isolates was tested. The distribution of BAL30072 MICs for the 61 strains tested was 7 isolates (11.5\%) at $0.004 \mu \mathrm{g} / \mathrm{mL}, 24$ isolates (39.3\%) at $0.008 \mu \mathrm{g} / \mathrm{mL}, 26$ isolates $(42.6 \%)$ at $0.016 \mu \mathrm{g} / \mathrm{mL}, 3$ isolates $(4.9 \%)$ at $0.032 \mu \mathrm{g} / \mathrm{mL}$ and 1 isolate $(1.6 \%)$ at 1 $\mu \mathrm{g} / \mathrm{mL}$ (Fig. 1). More than $93 \%$ of the isolates had MICs in the range $0.004-0.016 \mu \mathrm{g} / \mathrm{mL}$. Clinical and environmental strains exhibited similar susceptibility profiles. 


\subsection{Effects of chromosomally-encoded resistance determinants on BAL30072 susceptibility}

Very little is known about antibiotic resistance in B. pseudomallei and the only two mechanisms that have been characterised in some detail are the PenA $\beta$-lactamase $[10,11]$ and efflux $[12,13]$. Thus, the effects of $\beta$-lactamase and efflux on susceptibility to BAL30072 were tested. Like imipenem and meropenem, BAL30072 is only weakly hydrolysed by the chromosomally-encoded PenA class A $\beta$-lactamase (MICs for 1026b and the penA mutant Bp319 were $0.008 \mu \mathrm{g} / \mathrm{mL}$ and $0.002 \mu \mathrm{g} / \mathrm{mL}$, respectively) (Table 2). These observations were not due to lack of PenA expression in $1026 \mathrm{~b}$ because this strain expresses PenA and was highly resistant to amoxicillin and carbenicillin, two known PenA substrates. Deletion of penA in Bp319 resulted in significantly increased susceptibility to amoxicillin and carbenicillin, but not to BAL30072, ceftazidime, imipenem and meropenem. BAL30072 activity was not affected by efflux since the MICs observed with $1026 \mathrm{~b}$ and its $\triangle($ amrRABoprA) derivative Bp50 were the same $(0.008 \mu \mathrm{g} / \mathrm{mL})$.

\subsection{High-affinity iron transporters are not required for efficient BAL30072 activity}

The siderophore monosulfactam BAL30072 was designed to act like a 'Trojan horse' by exploiting iron uptake systems to gain access to its target. Presence of the catechol bioisosteric moiety is thought to facilitate ferric iron binding. The BAL30072-Fe $\mathrm{F}^{3+}$ complex could then potentially be actively transported across the outer membrane by a cognate outer membrane protein to gain access to the periplasm. The activity of BAL30072 against mutants Bp327, Bp338, Bp414 and Bp415 that were defective in malleobactin and/or pyochelin biosynthesis and transport was therefore tested. As all four mutants exhibited susceptibilities that were similar to the prototype strains $1026 \mathrm{~b}$ and $1710 \mathrm{~b}$, it was suggested that the high-affinity malleobactin and pyochelin receptors/transporters FmtA and FptA, respectively, are not required for efficient BAL30072 uptake. The lack of effect of impaired production or transport of malleobactin and pyochelin on BAL30072 is not unexpected owing to structural differences between siderophore moieties. Malleobactin is a hydroxamate siderophore [14] and pyochelin is a phenolate siderophore [15]. One would not expect the defect in a hydroxamate system to affect a catechol, and pyochelin is not good analogue of the catechol either.

\section{Conclusion}

It was previously noted that the unique pattern of penicillin-binding protein inhibition and bactericidal mode of action of BAL30072 confer potent in vitro activity against Gramnegative bacteria [5]. In this report, it was shown that BAL30072 has superior in vitro activity against $B$. pseudomallei compared with ceftazidime, meropenem and imipenem, as well as being rapidly bactericidal. It was not possible to attribute the superior activity of BAL30072 to facilitated cellular uptake via any of the currently characterised iron uptake systems of $B$. pseudomallei.

\section{Acknowledgments}

The authors wish to thank Vanaporn Wuthiekanun and Sharon Peacock (Faculty of Tropical Medicine, Mahidol University, Bangkok, Thailand) for providing the clinical and environmental B. pseudomallei strains used in this study.

Funding This work was supported by a grant (U54 AI065357) from the National Institute of Allergy and Infectious Diseases, National Institutes of Health, and a grant-in-aid of research by Basilea Pharmaceutica International Ltd. to HPS. 


\section{References}

[1]. Wiersinga WJ, van der Poll T, White NJ, Day NP, Peacock SJ. Melioidosis: insights into the pathogenicity of Burkholderia pseudomallei. Nat Rev Microbiol. 2006; 4:272-82. [PubMed: 16541135]

[2]. Dance, DAB. Melioidosis and glanders as possible biological weapons. In: Fong, W.; Alibek, K., editors. Bioterrorism and infectious agents: a new dilemma for the 21 st century. Springer Science and Business Media; New York, NY: 2005. p. 99-145.

[3]. Cheng AC, Currie BJ. Melioidosis: epidemiology, pathophysiology, and management. Clin Microbiol Rev. 2005; 18:383-416. [PubMed: 15831829]

[4]. Wuthiekanun V, Peacock SJ. Management of melioidosis. Expert Rev Anti Infect Ther. 2006; 4:445-55. [PubMed: 16771621]

[5]. Page MGP, Dantier C, Desarbre E. In vitro properties of BAL30072, a novel siderophore sulfactam with activity against multiresistant Gram-negative bacilli. Antimicrob Agents Chemother. 2010; 54:2291-302. [PubMed: 20308379]

[6]. Mushtaq S, Warner M, Livermore D. Activity of the siderophore monobactam BAL30072 against multiresistant non-fermenters. J Antimicrob Chemother. 2010; 65:266-70. [PubMed: 19996139]

[7]. DeShazer D, Brett P, Carlyon R, Woods D. Mutagenesis of Burkholderia pseudomallei with Tn5OT182: isolation of motility mutants and molecular characterization of the flagellin structural gene. J Bacteriol. 1997; 179:2116-25. [PubMed: 9079894]

[8]. Clinical and Laboratory Standards Institute. Methods for dilution antimicrobial susceptibility tests for bacteria that grow aerobically; approved standard. 7th ed.. CLSI; Wayne, PA: 2006.

Document M7-A7

[9]. Trunck LA, Propst KL, Wuthiekanun V, Tuanyok A, Beckstrom-Sternberg SM, BeckstromSternberg JS, et al. Molecular basis of rare aminoglycoside susceptibility and pathogenesis of Burkholderia pseudomallei clinical isolates from Thailand. PLoS Negl Trop Dis. 2009; 3:e519. [PubMed: 19771149]

[10]. Godfrey AJ, Wong S, Dance DA, Chaowagul W, Bryan LE. Pseudomonas pseudomallei resistance to $\beta$-lactam antibiotics due to alterations in the chromosomally encoded $\beta$-lactamase. Antimicrob Agents Chemother. 1991; 35:1635-40. [PubMed: 1718214]

[11]. Tribuddharat C, Moore RA, Baker P, Woods DE. Burkholderia pseudomallei class A $\beta$-lactamase mutations that confer selective resistance against ceftazidime or clavulanic acid inhibition. Antimicrob Agents Chemother. 2003; 47:2082-7. [PubMed: 12821450]

[12]. Moore RA, DeShazer D, Reckseidler S, Weissman A, Woods DE. Efflux-mediated aminoglycoside and macrolide resistance in Burkholderia pseudomallei. Antimicrob Agents Chemother. 1999; 43:465-70. [PubMed: 10049252]

[13]. Mima T, Schweizer HP. The BpeAB-OprB efflux pump of Burkholderia pseudomallei 1026b does not play a role in quorum sensing, virulence factor production, or extrusion of aminoglycosides but is a broad-spectrum drug efflux system. Antimicrob Agents Chemother. 2010; 54:3113-20. [PubMed: 20498323]

[14]. Alice AF, Lopez CS, Lowe CA, Ledesma MA, Crosa JH. Genetic and transcriptional analysis of the siderophore malleobactin biosynthesis and transport genes in the human pathogen Burkholderia pseudomallei K96243. J Bacteriol. 2006; 188:1551-66. [PubMed: 16452439]

[15]. Cox CD, Rinehart KL, Moore ML, Carter Cook J. Pyochelin: novel structure of an iron-chelating growth promoter for Pseudomonas aeruginosa. Proc Natl Acad Sci U S A. 1981; 78:4256-60. [PubMed: 6794030] 


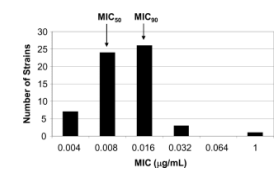

Fig. 1.

Minimal inhibitory concentration (MIC) distribution of BAL30072 in a panel of 61 Burkholderia pseudomallei strains. The $\mathrm{MIC}_{50}$ (MIC for $50 \%$ of the strains) and $\mathrm{MIC}_{90}$ (MIC for $90 \%$ of the strains) are indicated by arrows. 
Table 1

Bacterial strains used in this study

\begin{tabular}{|c|c|c|}
\hline Strain & Relevant genotype & Reference/source \\
\hline $1026 \mathrm{~b}$ & Prototype strain; clinical isolate & [7] \\
\hline $1710 \mathrm{~b}$ & Clinical isolate & S. Peacock \\
\hline $708 \mathrm{a}$ & $\begin{array}{l}\text { Clinical isolate; contains a }>140 \mathrm{~kb} \text { deletion that is fusing the equivalents of } 1710 \mathrm{~b} \text { BURPPS1710b_2155 and } \\
\text { BURPPS1710b_2054 genes; natural malleobactin biosynthesis gene cluster mutant }\end{array}$ & [9] \\
\hline Bp50 & $1026 \mathrm{~b}$ with $\triangle(a m r R A B$-oprA); AmrAB-OprA efflux pump mutant & This study \\
\hline Bp319 & $1026 \mathrm{~b}$ with $\triangle p e n A ;$ PenA $\beta$-lactamase deletion mutant & This study \\
\hline Bp327 & $1710 \mathrm{~b}$ with $\Delta(m b a S-m b a F)$; malleobactin biosynthesis gene cluster mutant & This study \\
\hline Bp338 & $\begin{array}{l}\text { 1710b with strain 708a } \triangle\left(B U R P P S 1710 b \_2155-B U R P P S 1710 b \_2054\right) \text {; malleobactin biosynthesis gene cluster } \\
\text { mutant }\end{array}$ & This study \\
\hline Bp414 & $1710 \mathrm{~b}$ with $\triangle f p t A ;$ pyochelin receptor mutant & This study \\
\hline Bp415 & $1710 \mathrm{~b}$ with $\Delta(m b a S-m b a F) \Delta f p t A$; malleobactin biosynthesis gene cluster and pyochelin receptor mutant & This study \\
\hline
\end{tabular}




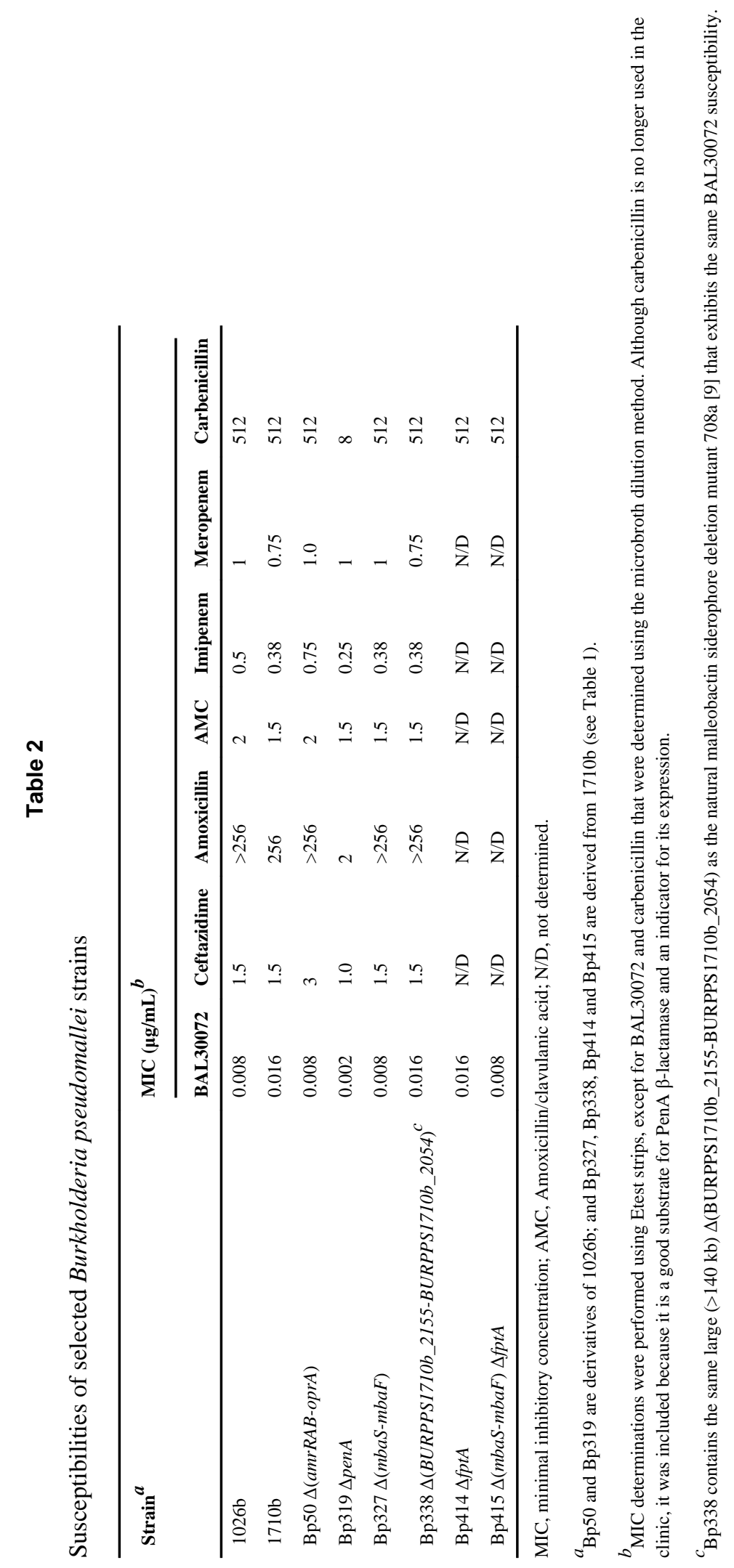

Int J Antimicrob Agents. Author manuscript; available in PMC 2011 August 1. 